\title{
Experimental demonstration of a hybrid III-V-on-silicon micro-laser based on resonant grating cavity mirrors
}

\author{
Yannick De Koninck, ${ }^{1, *}$ Fabrice Raineri, ${ }^{2,3}$ Alexandre Bazin, ${ }^{2}$ \\ Rama Raj, ${ }^{2}$ Gunther Roelkens, ${ }^{1}$ and Roel Baets ${ }^{1}$ \\ ${ }^{1}$ Department of Information Technology, Photonics Research Group, Ghent University - imec \\ Center for Nano-and Biophotonics (NB-photonics) \\ Sint-Pietersnieuwstraat 41, B-9000 Ghent-Belgium \\ ${ }^{2}$ Laboratoire de Photonique et de Nanostructures, CNRS-UPR20, Route de Nozay, 91460 Marcoussis, France \\ ${ }^{3}$ Université Paris Denis Diderot, 75205 Paris, France \\ *Corresponding author: yannick.dekoninck@intec.ugent.be
}

Compiled February 4, 2013

We present the first experimental demonstration of a novel class of hybrid III-V-on-silicon micro-lasers. We first show that by coupling a silicon cavity to a III-V waveguide, the interaction between the propagating mode in the III-V waveguide and the cavity mode in the silicon resonator results in high, narrow band reflection back into the III-V waveguide, forming a so-called resonant mirror. By combining two such mirrors and providing optical gain in the III-V wire in between these 2 mirrors, laser operation can be realized. This first optically pumped device measures $55 \mu \mathrm{m}$ by $2 \mu \mathrm{m}$, requires sub-mW threshold pump power and shows single mode laser emission with a side-mode suppression ratio of up to $39 \mathrm{~dB}$. (C) 2013 Optical Society of America

OCIS codes: $130.0250,140.3948,140.5960,250.5960$.

Over the past decade, silicon photonics has emerged as a major technology platform for photonic integrated components and circuits. Combining the mature fabrication processes inherited from CMOS with the high refractive index contrast between the silicon waveguide core and its cladding allows for unprecedented miniaturization of passive optical components. Unfortunately, silicon has an indirect bandgap, making it extremely difficult to use it as a laser gain medium. Amongst the different solutions that have been proposed to integrate coherent light sources directly onto the silicon die, one of the most promising approaches is to bond a slab of direct band-gap material (i.e. a III-V compound) onto the silicon die and perform a series of lithography and etching steps on that slab to obtain a laser cavity [1]. DBR- and DFB-lasers have been demonstrated $[1,2]$ on this platform where the laser mode is confined to the silicon layer and only its evanescent tail overlaps with the active III-V layer. This yields single-mode devices with high optical output, but the structures are large and require a fairly high threshold current $(>20 \mathrm{~mA})$. One way to reduce the threshold current is to scale down the size of the laser. Examples are III-V micro disk lasers [1], IIIV photonic-crystal cavity lasers [3] and photonic-crystal mirror VCSEL lasers [4]. The threshold pump power for these aforementioned micro-lasers is on the order of $1 \mathrm{~mW}$ or less. The lasing wavelength in these devices is either determined by the III-V processing or by the bonding layer thickness. In this work, we present the first experimental demonstration of a novel hybrid III$\mathrm{V}$ on silicon micro-laser. The proposed device is small, resulting in low threshold pump power, and offers intrinsic single-mode laser operation. Moreover the most critical device features are in the silicon layer, loosen-

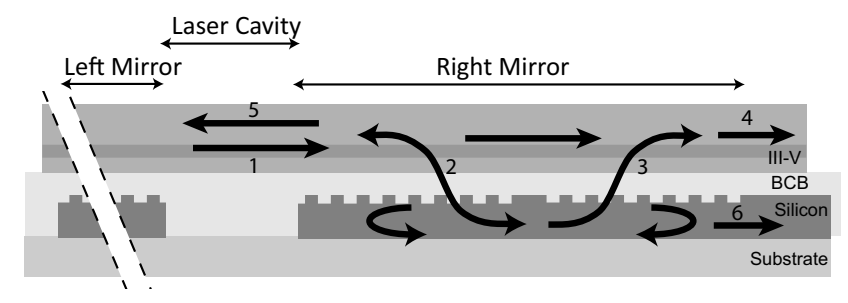

Fig. 1. Schematic side-view of the operation of the resonant mirror laser.

ing the lithography and etching requirements in the less mature III-V technology.

Like any other laser structure, the proposed design consists of 2 reflectors with the active laser cavity in between. The laser cavity is a III-V waveguide with no silicon structures underneath, so in this part of the laser the light is fully confined to that III-V waveguide. In each of the two mirror sections there is a silicon cavity underneath the III-V wire. Such a cavity can for example be a grating waveguide with a quarter-wavelength phase shifting section in the center to support a localized defect resonance [5]. The interaction between the guided waveguide mode in the III-V wire and the localized resonance in the silicon cavity underneath will result in high, narrow-band reflection back into the III$\mathrm{V}$ waveguide, hence the name resonant mirror. Figure 1 shows a schematic side-view of the laser. Light is generated and amplified in the III-V wire. As the light approaches the end of the III-V wire ( 1 in figure 1), a small part of the light couples to the silicon cavity underneath (2). For wavelengths close to the silicon cavity resonance wavelength, power will build up inside the silicon cav- 


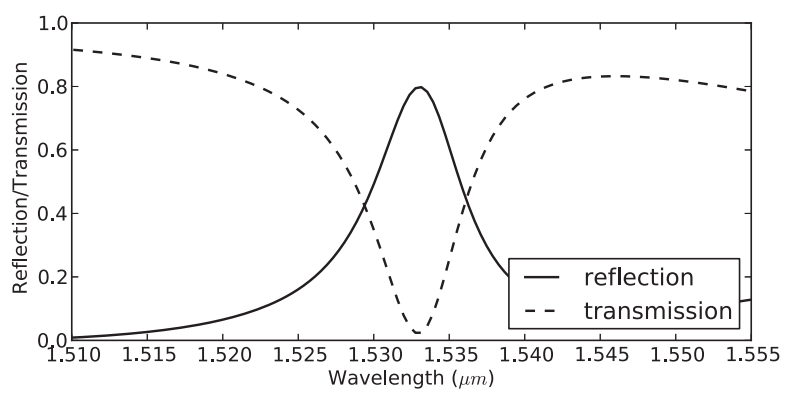

Fig. 2. Simulated reflection and transmission spectrum of a resonant mirror.

ity. Eventually the energy stored in the silicon cavity is high enough such that a significant amount of optical power will couple back into the III-V wire. The light coupling back co-directionally to the incident light (3) will interfere destructively to the latter, resulting in zero transmission (4). On the other hand, the light coupling back counter-directionally to the incident light (2) will provide feedback into the III-V wire (5), required to establish laser operation. Light can easily be extracted to a silicon output waveguide by tapping a small amount of the power built-up in the silicon cavity to an external waveguide (6). A cavity coupled to the laser mesa to serve as a narrow band reflector was used in the past to reduce the laser line width [6]. More recently, the same principle was also used to design narrow-band passive optical filters [7].

Because the power-buildup only occurs close to the silicon cavity resonance wavelength, the reflection bandwidth of this type of mirror is relatively narrow $(<$ $10 \mathrm{~nm}$ ). Since the reflection of such a mirror can be very high and the laser mode is confined to the III-V wire, which maximizes the modal gain, the length of the III-V wire can be reduced to make sure only one longitudinal resonance of the III-V cavity lies within the reflection bandwidth of the mirror, yielding an intrinsically singlemode laser with a short length.

Figure 2 shows the reflection and transmission spectrum of a resonant mirror simulated using 3D-FDTD. In this particular mirror, the silicon cavity is a $1.6 \mu \mathrm{m}$ wide, $220 \mathrm{~nm}$ high strip waveguide with $70 \mathrm{~nm}$ deep periodic grating corrugations. It consists of 60 periods $(285 \mathrm{~nm}$ period pitch) with a quarter-wavelength defect in the middle to support a resonance near the grating's Bragg wavelength. The III-V wire above is $1.6 \mu \mathrm{m}$ wide and consists of an $80 \mathrm{~nm}$ thick InGaAsP (Q1.55) bulk active layer, sandwiched between two InP cladding layers of $10 \mathrm{~nm}$ (below InGaAsP) and $120 \mathrm{~nm}$ (above InGaAsP) respectively. The vertical spacing between the silicon layer and III-V layer is $240 \mathrm{~nm}$.

The simulated spectrum in figure 2 shows that, at the silicon cavity's resonance wavelength $(1533 \mathrm{~nm})$, the structure reflects $80 \%$ of the incoming power back into the III-V waveguide. At that same wavelength, virtu-

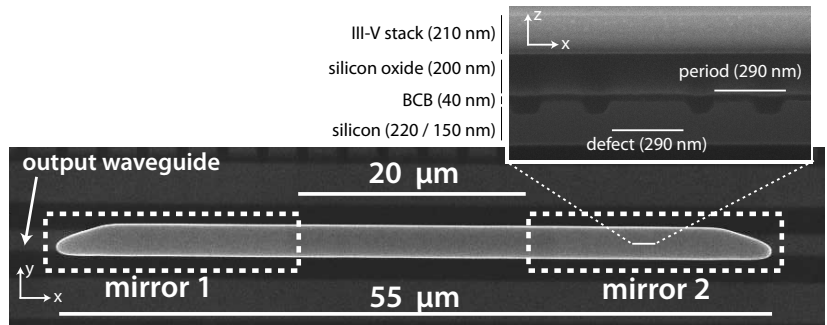

Fig. 3. SEM top-image of a finished device. inset: mirror cross-section at grating defect.

ally no power $(2.5 \%)$ is transmitted through the III-V waveguide. Most of the remaining $17 \%$ is directed to the output silicon waveguide. The full-width half-max bandwidth of the reflection peak is $7 \mathrm{~nm}$.

The optimal distance between the two mirrors in the laser configuration is determined by the reflection bandwidth of the mirror. To guarantee single mode operation, the FSR of the laser's longitudinal modes should be at least on the order of the reflection bandwidth $(7 \mathrm{~nm})$, so the spacing between the mirrors should be sufficiently small. On the other hand, this mirror's peak reflectivity is only $80 \%$, so the III-V waveguide should be sufficiently long to ensure that laser threshold can be reached with a reasonable pump power. Considering these two conditions, calculations show that a good value for the spacing between the silicon mirrors is $20 \mu \mathrm{m}$, resulting in a laser cavity FSR of around $6 \mathrm{~nm}$.

The device was fabricated starting from a SOI die processed in a $200 \mathrm{~mm}$ pilot line through the ePIXfab multi project wafer service [8] and a $210 \mathrm{~nm}$ thick III-V stack on an InP substrate. The composition of the III-V stack is as described above. The SOI die and the III-V die are bonded together using DVS-BCB adhesive bonding [1]. The resulting spacing between the top of the SOI waveguide layer and the bottom of the III-V stack measures $240 \mathrm{~nm}\left(200 \mathrm{~nm} \mathrm{SiO}_{2}+40 \mathrm{~nm}\right.$ DVS-BCB). After removing the InP substrate, the III-V mesa is defined using contact lithography and an inductively coupled plasma etch.

Figure 3 shows a top-view SEM image of a finished device. The III-V waveguide masks the silicon cavities in the picture but the white dashed boxes indicate the location and longitudinal extent of both silicon grating cavities. In this particular case, both mirrors are identical and both have a silicon output waveguide. Each laser is $55 \mu \mathrm{m}$ long and $2 \mu \mathrm{m}$ wide. The silicon cavities each consist of 60 periods with a defect (quarter-wave shift) in the center. Although devices with different grating periods were fabricated, the grating period of this particular device is $285 \mathrm{~nm}$, so each silicon cavity measures around $17 \mu \mathrm{m}$. The silicon cavities are spaced by $20 \mu \mathrm{m}$. The tapered edges of the III-V wire are intentionally patterned to avoid back-reflection from the III-V waveguide facets. Both silicon output waveguides each lead to a grating fiber-coupler. The inset in figure 3 is a FIB cross-section 


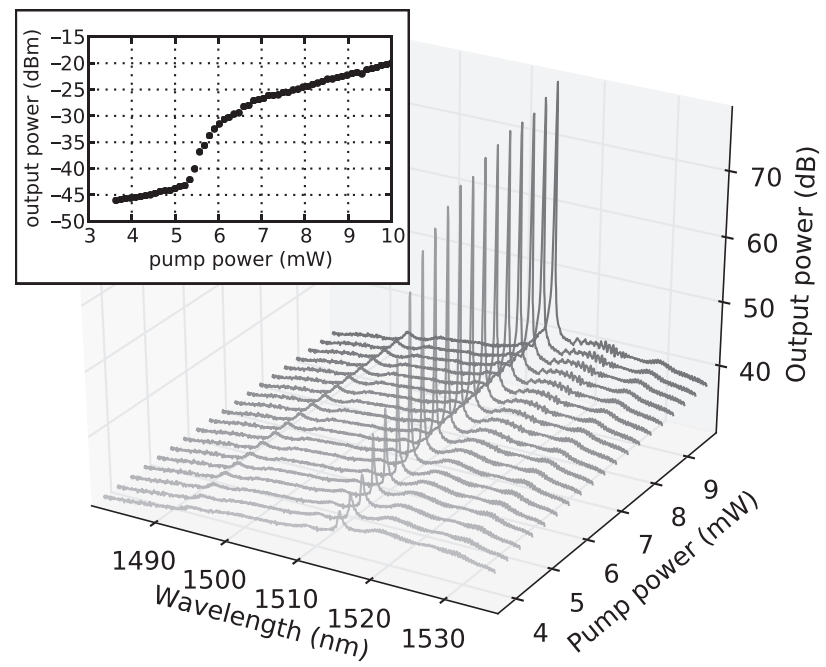

Fig. 4. Measured laser output-spectrum for different values of the pump-power. inset: Estimated laser output power as a function of the pump power.

of one of the mirrors showing the quarter-wavelength defect in the silicon grating.

The devices were measured at room temperature by illuminating each device individually from the top with an $800 \mathrm{~nm}$ DFB pump laser. This pump laser was modulated to obtain $10 \mathrm{~ns}$ pulses with $10 \mathrm{~mW}$ peak power and a repetition rate of $1.5 \mu \mathrm{s}$. Using an objective and a cylindrical lens, the shape of the illuminating spot was adjusted to match the shape of the III-V waveguide to be pumped. The output of the resonant-mirror laser is coupled to a silicon waveguide which guides it towards a grating coupler. From there, a cleaved fiber directs the laser output to a spectrometer equipped with a nitrogen cooled InGaAs line camera to measure the spectral power distribution of the laser output.

The laser output spectrum was measured for different values of the pump power and the result is shown in figure 4 . This graph clearly shows the intrinsic single-mode nature of this device: there is no competition between different longitudinal modes, not even at low pump power values, because the resonant mirrors are designed to reflect only 1 longitudinal laser-mode. To obtain the relationship between the laser output power and the pump power (the light-light curve for optically pumped devices), the measured output spectra in figure 4 are integrated for each value of the pump power individually. The results are shown in the inset of figure 4. This graph very clearly shows the lasing threshold between $5 \mathrm{~mW}$ and $6 \mathrm{~mW}$ pump power. Note that the $\mathrm{x}$-axis values are the total incident pump laser power. Because only a fraction of this power is absorbed by the active layer of the III-V waveguide, we estimate that the actual power required to reach laser threshold is well below $1 \mathrm{~mW}$. The laser peak output power is conservatively estimated to be $10 \mu W$ and is limited by the available pump power. The duration of the pump-pulse was increased and laser oper-

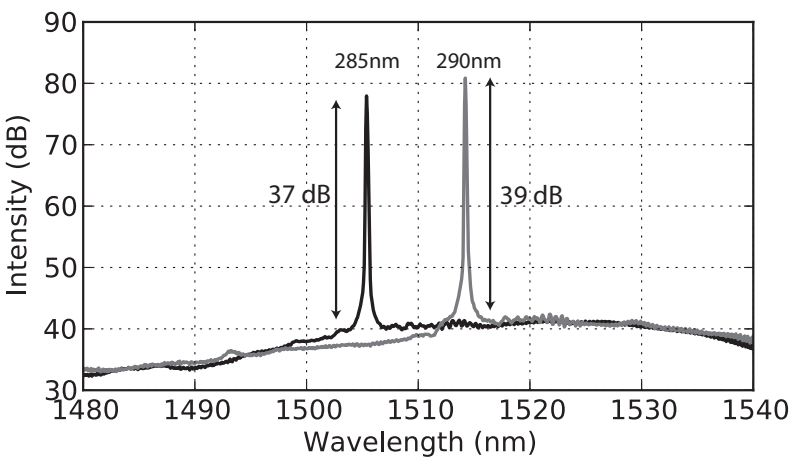

Fig. 5. Measured output spectrum of two different lasers: one with a $285 \mathrm{~nm}$ grating pitch and one with a $290 \mathrm{~nm}$ grating pitch.

ation continued up until a pulse duration of $1.2 \mu \mathrm{s}$ (with a repetition rate of $1.5 \mu \mathrm{s}$, yielding an $80 \%$ duty-cycle). The failure to operate in a continuous wave regime is most-likely due to self-heating but this can be improved in future designs by optimizing the thermal impedance of the device.

To investigate the spectral properties of the laser, consider figure 5. This graph shows the measured output spectrum for 2 different devices: one with a grating period of $285 \mathrm{~nm}$ and the other one with a grating period of $290 \mathrm{~nm}$. Apart from the grating period both devices are identical. This proves that the lasing wavelength is defined by the resonant wavelength in the silicon cavity. The side-mode suppression ratio (SMSR) is very high in both cases (37 $\mathrm{dB}$ and $39 \mathrm{~dB}$ respectively).

The authors acknowledge partial support from IWT through the SBO-Glucosens project as well as from ERC through the InSpectra project. Yannick De Koninck thanks the research foundation Flanders (FWO) for a research grant.

\section{References}

1. G. Roelkens, L. Liu, D. Liang, R. Jones, A. Fang, B. Koch, and J. Bowers, Laser \& Photonics Reviews 4, 751 (2010).

2. S. Stankovic, R. Jones, M. N. Sysak, J. M. Heck, G. Roelkens, and D. Van Thourhout, IEEE Photonics Technology Letters 24, 2155 (2012).

3. Y. Halioua, A. Bazin, P. Monnier, T. J. Karle, G. Roelkens, I. Sagnes, R. Raj, and F. Raineri, Optics express 19, 9221 (2011).

4. C. Sciancalepore, B. B. Bakir, X. Letartre, J. Harduin, N. Olivier, C. Seassal, J.-m. Fedeli, and P. Viktorovitch, IEEE Photonics Technology Letters 24, 455 (2012).

5. Y. De Koninck, G. Roelkens, and R. Baets, in IEEE Photonics Society Annual Meeting 2010 (2010).

6. H. A. Haus and Y. Lai, Journal of Lightwave Technology 9, 754 (1991).

7. K. Debnath, K. Welna, M. Ferrera, K. Deasy, D. G. Lidzey, and L. OFaolain, Optics Letters 38, 154 (2013).

8. The Silicon Photonics Platform-ePIXfab http://www.epixfab.eu, "ePIXfab", (2012). 


\section{Informational Fourth Page}

In this section, please provide full versions of citations to assist reviewers and editors (OL publishes a short form of citations) or any other information that would aid the peer-review process.

\section{References}

1. G. Roelkens, L. Liu, D. Liang, R. Jones, A. Fang, B. Koch, and J. Bowers, Laser \& Photonics Reviews 4, 751 (2010).

2. S. Stankovic, R. Jones, M. N. Sysak, J. M. Heck, G. Roelkens, and D. Van Thourhout, IEEE Photonics Technology Letters 24, 2155 (2012).

3. Y. Halioua, A. Bazin, P. Monnier, T. J. Karle, G. Roelkens, I. Sagnes, R. Raj, and F. Raineri, Optics express 19, 9221 (2011).

4. C. Sciancalepore, B. B. Bakir, X. Letartre, J. Harduin, N. Olivier, C. Seassal, J.-m. Fedeli, and P. Viktorovitch, IEEE Photonics Technology Letters 24, 455 (2012).

5. Y. De Koninck, G. Roelkens, and R. Baets, in IEEE Photonics Society Annual Meeting 2010 (2010).

6. H. A. Haus and Y. Lai, Journal of Lightwave Technology 9, 754 (1991).

7. K. Debnath, K. Welna, M. Ferrera, K. Deasy, D. G. Lidzey, and L. OFaolain, Optics Letters 38, 154 (2013).

8. The Silicon Photonics Platform-ePIXfab http://www.epixfab.eu, "ePIXfab", (2012). 